\title{
Surgical Management of Complicated Colon Cancer
}

\author{
Steven Lee-Kong, $\mathrm{MD}^{1} \quad$ David Lisle, $\mathrm{MD}^{2}$ \\ 1 Department of Surgery, CUMC, New York Presbyterian Hospital, \\ Columbia University, New York, New York \\ 2 Department of General Surgery, New York Presbyterian Hospital, \\ Columbia University, New York, New York
}

\begin{abstract}
Address for correspondence Steven Lee-Kong, MD, Department of General Surgery, 161 Fort Washington Avenue, New York, New York 10032 (e-mail: sal116@cumc.columbia.edu).
\end{abstract}

Clin Colon Rectal Surg 2015;28:228-233.

\author{
Abstract \\ Keywords \\ - colonic stent \\ - large bowel \\ obstruction \\ - colon cancer \\ - multivisceral \\ resection \\ - colon perforation
}

The management of complicated colon cancer (locally invasive, obstructed, or perforated cancers) can pose diagnostic and therapeutic challenges to surgical management. Adherence to traditional surgical oncologic principles must often be balanced with the patients' clinical presentation and other parameters. While the goal of an R0 (no residual microscopic disease) resection must always be kept in mind, situations sometimes arise which can make this difficult to achieve. Recognition of complicated disease and availability of varied therapeutic modalities is important to ensure favorable patient outcomes. This review will discuss the surgical management of complicated colon cancer, with special focus on locally advanced disease.
Colon cancer is the third most common cancer in both men and women in the United States, and accounts for over 57,000 deaths annually. ${ }^{1}$ Prognosis is most accurately determined based on American Joint Commission on Cancer (AJCC) stage, as determined after extirpative surgery. The surgical management of colon cancer has undergone an impressive transformation over the past two decades, as has our ability to evaluate and treat patients with advanced disease. Up to $20 \%$ of newly diagnosed colon cancer patients initially present with locally advanced disease, with perforation of the colon, invasion of adjacent structures, large bowel obstruction, and/or significant hemorrhage. These patients often present with symptoms corresponding to the primary tumor site (i.e., obstruction in the narrow caliber left colon, back pain in tumors penetrating the retroperitoneum, urinary symptoms in patients with colovesical fistulae). This review aims to highlight treatment options available for patients with locally advanced colon cancer, and to review the data supporting these avenues of treatment. We will not review the management of colorectal liver metastases or peritoneal carcinomatosis.

Issue Theme Colon Cancer; Guest Editor: Garrett M. Nash, MD, MPH, FACS, FASCRS

\section{Management of Locally Invasive Colon Cancer (Multivisceral Resection)}

Patients presenting with locally advanced colon cancer, with tumors invading other organs, can present both a diagnostic and therapeutic challenge to surgeons. These patients can be offered multivisceral resection (MVR; resection of colonic segment bearing the cancer en bloc with secondarily involved organ). The first series of such resection was reported by Sugarbaker in $1946 .{ }^{2}$ With the improvements in cross-sectional imaging, these patients can often be identified prior to attempt at surgical resection (-Fig. 1). This is critical in terms of surgical treatment planning, as teams skilled in MVR may be required to ensure good outcomes (both functionally and oncologically). Surgeons who are comfortable performing en bloc resections of the bladder, duodenum, pancreas, abdominal wall, or spleen are occasionally required for MVRs.

The most crucial consideration in the assessment of a patient for an MVR is the ability to achieve an R0 resection. Inability to achieve negative margins portends a poorer prognosis and is of little survival advantage. Lehnert et al reviewed, over a 16-year period, 201 (139 colon cancer, 62

Copyright (c) 2015 by Thieme Medical Publishers, Inc., 333 Seventh Avenue, New York, NY 10001, USA. Tel: +1(212) 584-4662. 


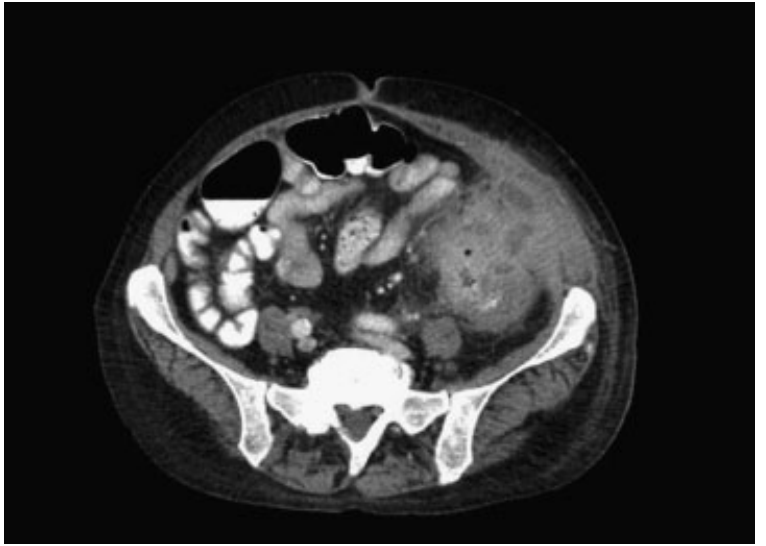

Fig. 1 Locally advanced colon cancer involving the abdominal wall.

rectal cancer) patients who presented with locally invasive colorectal cancers. ${ }^{3}$ The distribution of cancers treated with MVR includes small bowel, urinary bladder, and abdominal wall involvement. Actual tumor infiltration (as opposed to inflammatory adhesions) was evident in 48,50 , and $8 \%$ of these organs, respectively. For colon cancer patients, morbidity after MVR was $28 \%$ and the mortality rate was $9.4 \%$. The most important association with overall survival was the ability to achieve an R0 resection (-Fig. 2), and survival was similar to patients undergoing conventional surgery for stage-matched colon cancers not requiring MVR (-Fig. 3).

A similar study by Croner et al evaluated 174 patients treated with MVR for colon cancer between 1978 and 2002. ${ }^{4}$ Similar to the Lehnert study, the majority of colon cancers were of the sigmoid colon, and the most common additional organs involved were small intestine, urinary bladder, and abdominal wall (32.6, 27.0, and $15.5 \%$, respectively). Overall complication rate was $25.8 \%$, similar to the Lehnert study. Cancer-related survival was excellent if an R0 resection was achieved (-Fig. 4).

Many surgeons still consider the need for MVR for colon cancer a contraindication for laparoscopy; nevertheless,

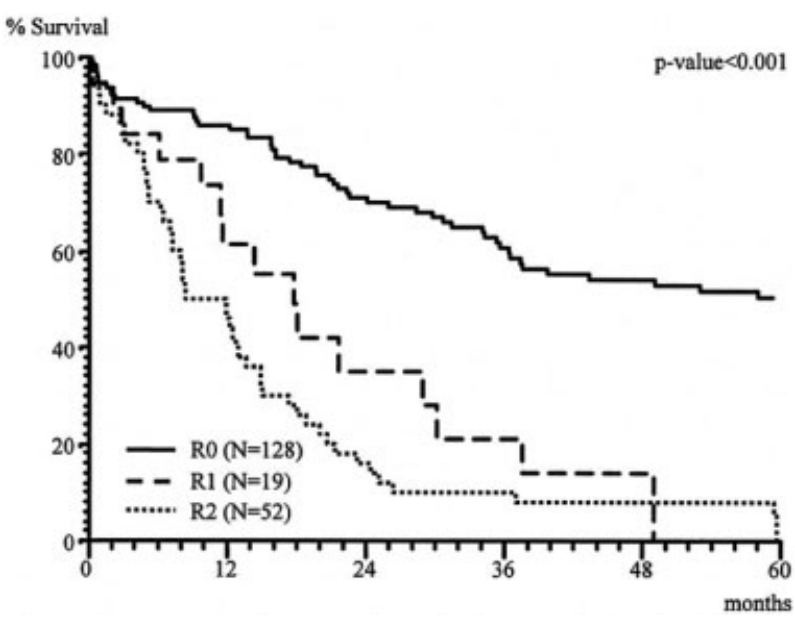

Fig. 2 Overall survival after multivisceral resection.

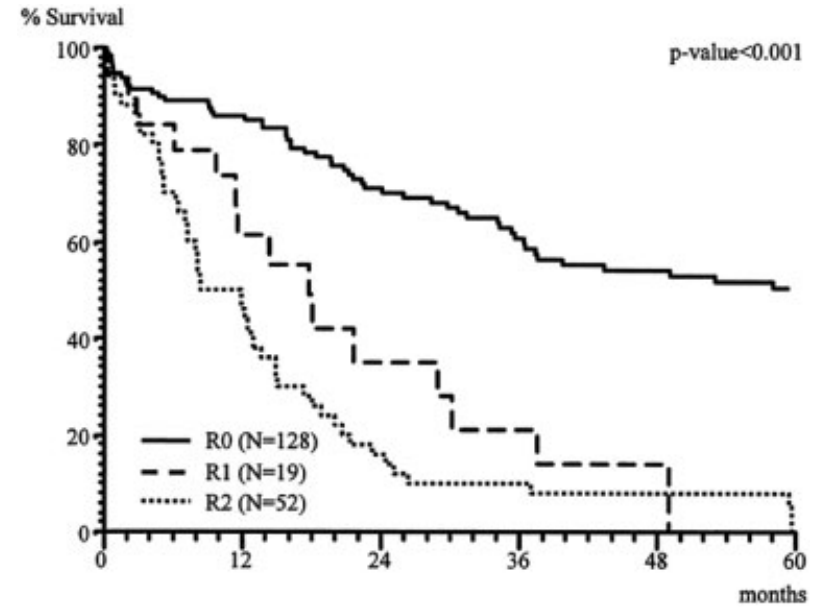

Fig. 3 Overall survival after conventional versus multivisceral RO resection (only T3/T4 tumors).

increasing experience with minimally invasive approaches has made this more acceptable. Initial concerns about oncologic adequacy, as well as the technical demands of these complex resections, have made some surgeons reluctant to undertake these operations. Taken on a case-by-case basis, appropriately selected patients can be considered for a laparoscopic approach.

A recent case-matched study by Vignali et al suggests that a laparoscopic approach to patients with locally advanced colon cancer can be safe and effective. ${ }^{5}$ The authors identified 70 patients, over a 10 -year period, who underwent laparoscopic MVR for primary colon cancer, and matched them with 70 patients who underwent open surgery for the same. Patients undergoing laparoscopic MVR experienced less blood loss, lower blood product transfusion requirement, and shorter length of stay, but longer operative time when compared with open surgery. There were no differences in readmission rate, adequacy of lymphadenectomy, or overall survival. Despite the limitations of this retrospective cohort study, it does appear that in carefully selected patients, MVR

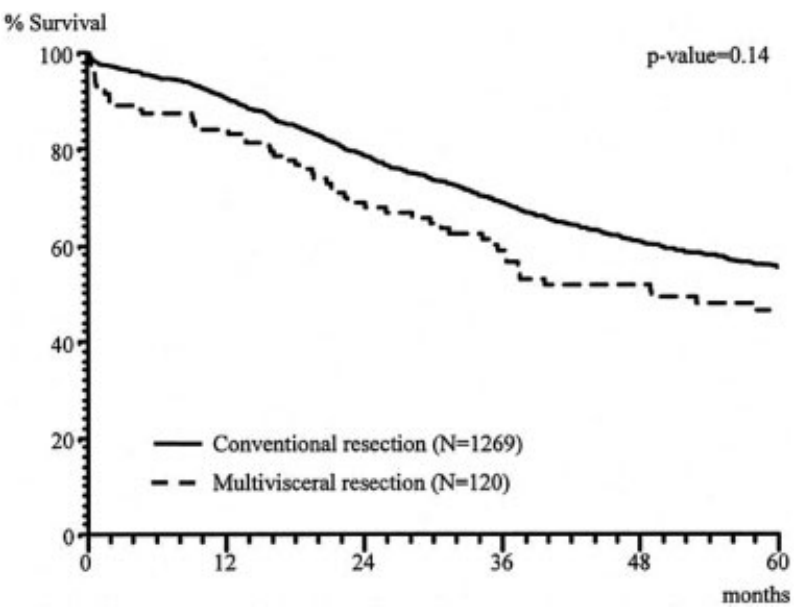

Fig. 4 Cancer-related survival after multivisceral RO versus R1, R2 resection. 
can be performed safely with the benefits of a minimally invasive approach and without compromising oncologic outcome. Larger prospective studies are needed to validate these conclusions.

There are several small reports illustrating the utility of neoadjuvant chemotherapy or chemoradiation for the management of locally advanced colon cancer. Both Hallet et al and Cukier et al describe small numbers of patients receiving these treatments prior to MVR. ${ }^{6,7}$ These authors report a $100 \% \mathrm{R} 0$ resection rate as well as excellent 3-year and overall and diseasefree survival (85.9 and $73.7 \%$, respectively). While the number of patients in these studies is quite small, it does suggest potential role for neoadjuvant treatment prior to MVR.

In summary, MVR can be safely performed for locally advanced/invasive colon cancers (and in carefully selected patients, minimally invasively). The ability to achieve an R0 resection is the greatest determinate of outcome, with improved survival over incompletely resected tumors. Careful attention to preoperative imaging and availability of specialized assistance (when required) are essential during operative planning.

\section{Management of Obstructing Colon Cancer}

Up to $30 \%$ of newly diagnosed colon cancers present with acute intestinal obstruction, particularly in the case of leftsided tumors. ${ }^{8}$ The majority of obstructing lesions are in the sigmoid or rectosigmoid colon. Traditional surgical management included diverting stoma creation, extended (subtotal) colectomy with anastomosis or a resection, and end stoma creation. These can be done in either two or three stages. Approximately $30 \%$ of patients who have diverting stomas created never go on to have the stoma reversed, incurring significant long-term morbidity. ${ }^{9}$ The past several years have seen the development of techniques to relieve malignant colonic obstructions with the use of laser, argon plasma coagulation, and cryosurgery. ${ }^{10-12}$ The most promising and readily available advance in the management of patients with obstructing colon cancers is the endoluminal colonic stent.

For proximal colon lesions, right colectomy with a primary ileocolic anastomosis is considered the surgical treatment of choice (-Fig. 5). Even in the emergency setting, primary anastomosis is associated with a less than $5 \%$ risk of anastomotic leakage. ${ }^{13}$ Newer stent delivery devices now make selected right colonic lesions accessible to endoluminal stenting, and can be utilized in select cases not suitable for surgical resection.

For distal obstructing lesions, segmental colectomy with primary anastomosis or subtotal colectomy with an ileocolic or ileorectal anastomosis can be considered. Segmental colectomy is associated with an improved functional result when compared with subtotal colectomy (i.e., fewer bowel movements); however, anastomosing dilated proximal colon to more normal caliber distal colon or rectum can be worrisome and an occult proximal neoplasm may be left untreated. The data regarding colonic surgery in the absence of a mechanical bowel preparation are conflicted, ${ }^{14}$ and some may consider on-table colonic lavage in this setting. With a

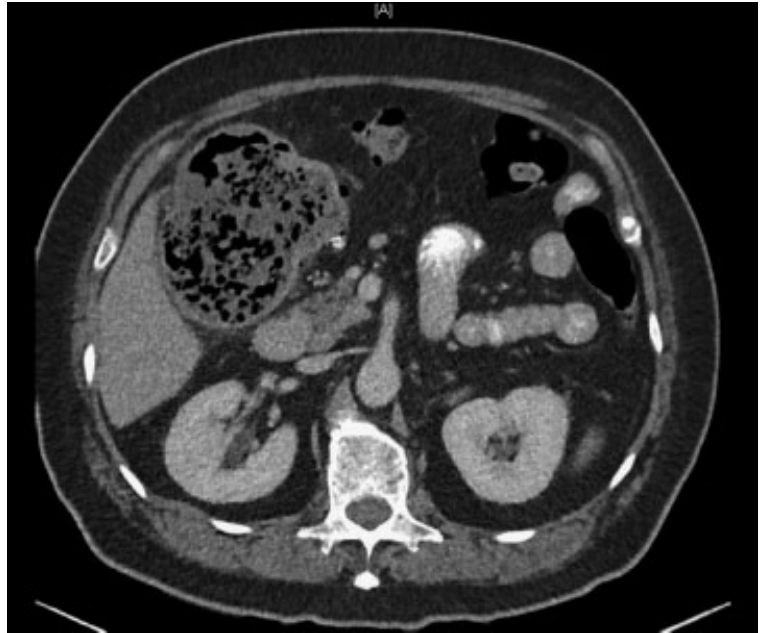

Fig. 5 Colon cancer with right colon obstruction.

subtotal colectomy, ileocolic anastomoses are believed to result in a lower anastomotic leakage rate. ${ }^{15}$

First described in 1996, Dohmoto et al reported their experience of endoluminal stenting of obstructing rectal cancers in 19 patients with unresectable or metastatic disease. ${ }^{16}$ These patients were first treated with either endoscopic dilation or laser-assisted recanalization of the gastrointestinal lumen. The endoprosthetics used were first "modified plastic tubes" in the first few patients followed by self-expanding nitinol mesh stents or nickel titanium coil stents (-Figs. 6 and 7). All patients were successfully stented and had near immediate relief of obstructive symptoms. The authors reported seven complications, most being stent dislocation and occlusion. This early report demonstrated the feasibility of endoluminal stenting in select patients.

Colonic stents can be used either as primary treatment for patients with obstructing tumors not amenable to resection or as a bridge to surgical resection. For patients who are

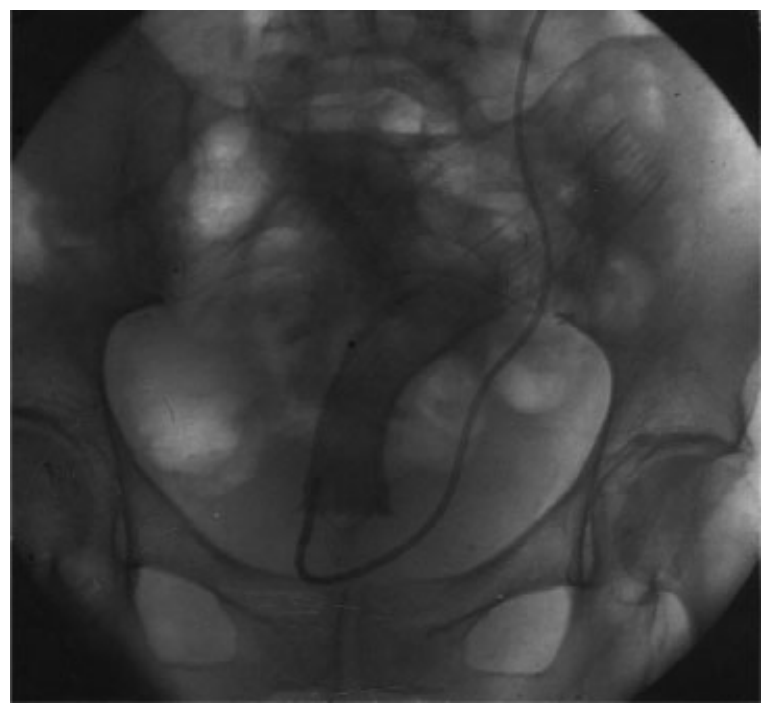

Fig. 6 Radiograph showing full expansion of a self-expanding mesh stent in stenotic rectal cancer. 


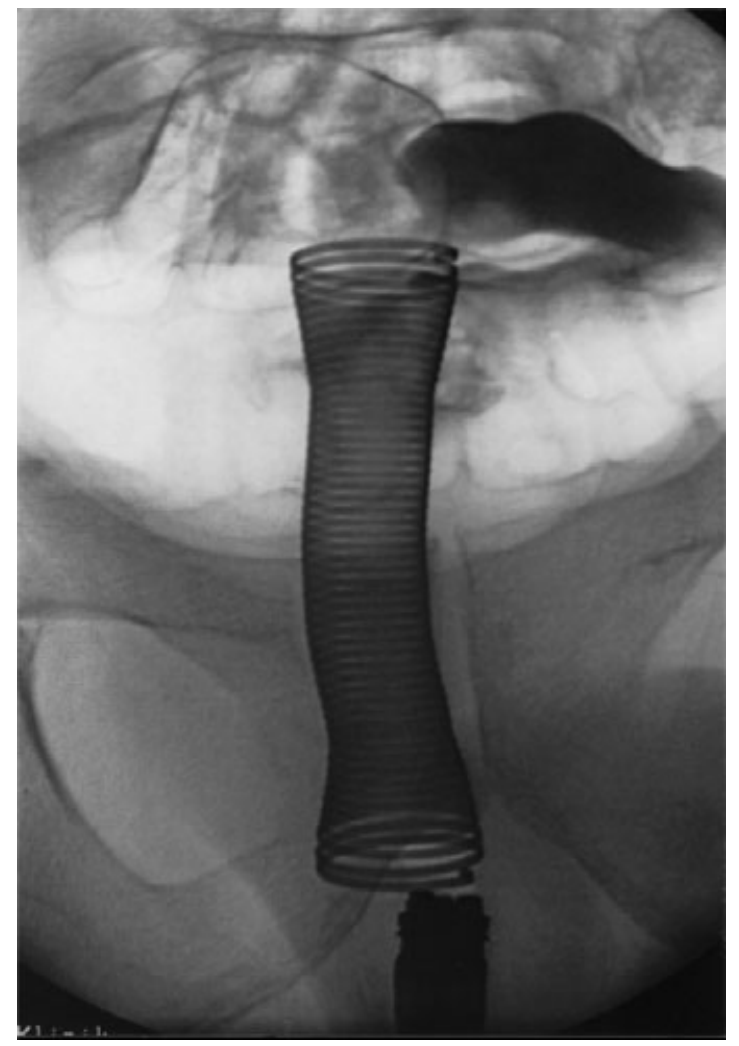

Fig. 7 Obstructing rectal cancer: after implantation of an endocoil stent luminal patency is demonstrated by unimpeded flow of contrast agent through the stent.

"bridged" with endoluminal stenting, resection can be considered once the proximal colon has been adequately decompressed and, at surgeon preference, the patient can undergo a mechanical bowel preparation. Numerous randomized controlled trials have been conducted to investigate the role of endoluminal stenting as a bridge to surgery, with several of them closing prematurely due to elevated morbidity in the stenting groups. ${ }^{17-22}$ van Hooft et al conducted a large, multicenter randomized trial investigating the benefit of using endoluminal stenting for these patients. ${ }^{18}$ They randomized 98 patients with acute left-sided malignant colon obstructions to receive either emergent surgery or endoluminal stent placement. Interim analyses demonstrated increased 30-day morbidity in the stented group (absolute risk increase of 0.19). The data safety monitoring committee ended this study prematurely due to the above findings.

Van den Berg et al reviewed their experience with 48 patients who received endoscopic stent placement for malignant colon obstruction. ${ }^{23}$ While technical and short-term clinical success rates were high (91 and 85\%, respectively), stent-related morbidity was $38 \%$ and mortality was $13 \%$, and the one year stent-patency rate was $50 \%$. The authors highlight that while endoscopic stents are successful at relieving acute obstructions, the price paid may be significant stent-related morbidity and poor long-term patency rates.

Effective long-term palliation of obstructing left colon cancers has been demonstrated by other groups, however. $^{23,24}$ Thankfully, it is the minority of patients with meta- static colorectal cancer who need palliation. ${ }^{23}$ Poultsides et al reviewed the experience of patients with stage IV colorectal cancer receiving "modern" chemotherapy at Memorial Sloan-Kettering Cancer Center. ${ }^{25}$ Only $11 \%$ of patients developed a primary tumor complication while receiving chemotherapy, with 11 out of 26 patients receiving an endoluminal stent for obstruction (-Fig. 8). These results have been corroborated by others as well.

The management of obstructing left-sided colon cancers should be individualized based on patient factors, experience of the surgeon or gastroenterologist with less invasive approaches, and practice setting. In circumstances where emergent surgery is likely to require stoma creation, initial treatment with an endoluminal stent may allow for future one-stage surgery and prevent stoma creation (with its associated morbidity and decrement in quality of life). For patients on whom endoluminal approaches are not feasible, upfront surgical resection and/or fecal diversion are more appropriate.

\section{Management of Perforated Colon Cancer}

Perforated colon cancer presents a unique challenge to the surgeon. Adherence to surgical oncologic principles is often outweighed by the emergent nature of surgery for these patients. Controlling sepsis, managing shock, and achieving "source control" in these sometimes unstable patients can take precedent over achieving an $\mathrm{R} 0$ resection or adequate lymphadenectomy in these potentially unstable patients. Data are somewhat limited regarding the outcomes of patients undergoing emergent surgery for colon cancer perforation, and most reports are small single-institutional series. Most reports, however, detail poor outcome in patients who present with perforation. ${ }^{26}$ As these patients often present emergently, prospective (and certainly randomized) data will be difficult to ever obtain.

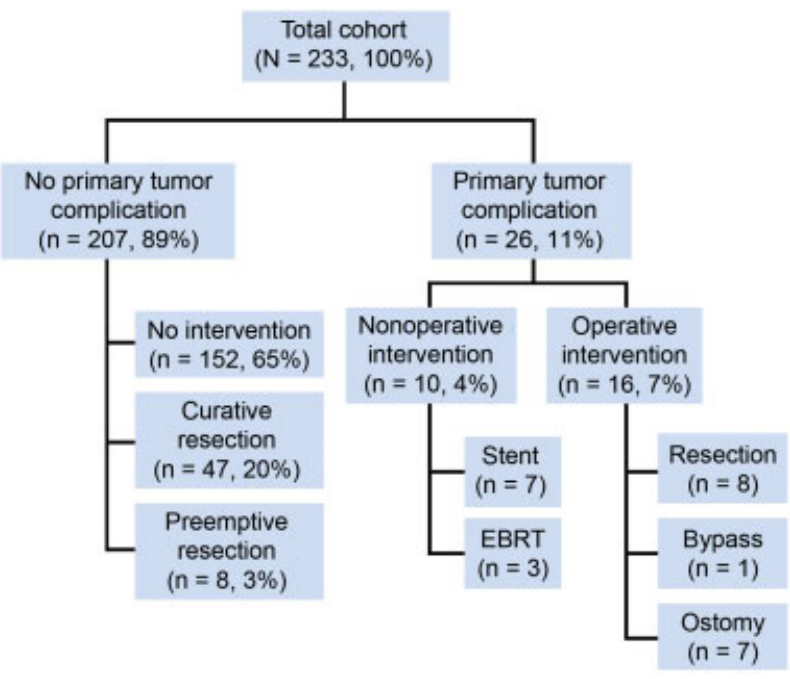

Fig. 8 Development of primary tumor complication in patients with stage IV colorectal cancer while receiving chemotherapy. 
Biondo et al reported their experience of 38 patients who presented with perforated colon cancer, and compared their outcome to 155 patients during the same time period who presented with obstructing tumors. ${ }^{27}$ All patients underwent curative-intent surgery. Most tumor perforations were at or near the site of the primary tumor, with $3 / 38$ patients manifesting perforation in the proximal, distended colon. The authors report no difference in complication rates, overall survival, recurrence rates, or use of adjuvant chemotherapy between the two groups.

Zielinski et al examined patients who presented with perforated colon cancers, identifying patients who had either a free perforation (feculent or purulent peritonitis) or contained perforation (abscess or fistula formation). ${ }^{28}$ Patients with free perforation had a significantly higher mortality when compared with patients with contained perforation (19 vs. $0 \%$, respectively; $p=0.038$ ), and overall survival was significantly worse (24 vs. $62 \% ; p=0.003$ ) (-Fig. 9), as well as 5-year disease-free survival (15 vs. $53 \% ; p<0.001$ ).

Patients who present with intestinal perforation from colon cancer can be challenging to manage. The importance of an adequate oncologic resection must be weighed against the emergent nature of the procedure and rapid control of ongoing sepsis and restoration of hemodynamic parameters for patients in extremis.

\section{Summary}

Patients who present with complicated colon cancers often pose both diagnostic and therapeutic dilemmas for surgeons. Care plans must be individualized based on patient presentation, comorbid conditions, and urgency of intervention. When feasible, we recommend a multidisciplinary approach to patients presenting with complicated disease (usually including surgeons, medical oncologists, interventional endoscopists, and radiologists). Early referral to a medical oncologist postoperative is also crucial, as adjuvant chemotherapy may prove beneficial (even in node-negative disease).

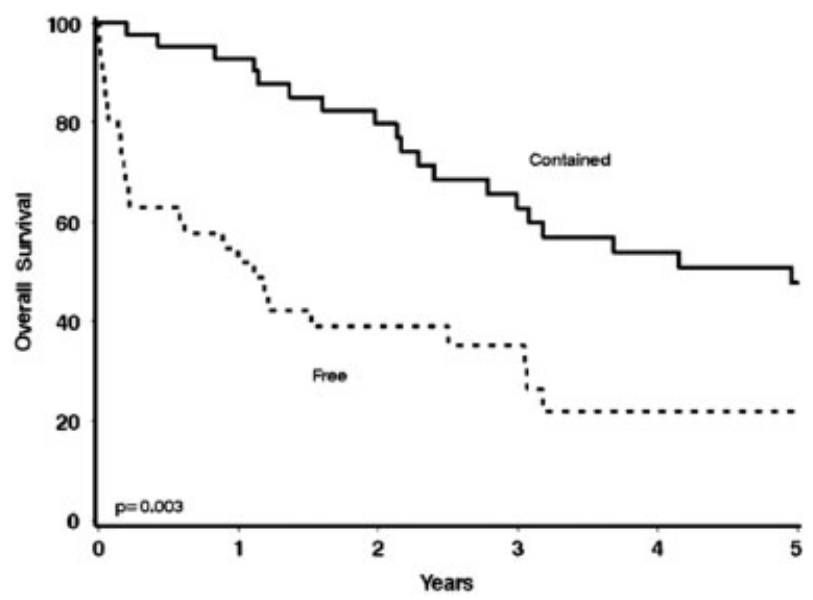

Fig. 9 Overall survival in patients with contained perforated colorectal cancer versus free perforation.

\section{References}

1 Siegel R, Ma J, Zou Z, Jemal A. Cancer statistics, 2014. CA Cancer J Clin 2014;64(1):9-29

2 Sugarbaker ED. Coincident removal of additional structures in resections for carcinoma of the colon and rectum. Ann Surg 1946; 123:1036-1046

3 Lehnert T, Methner M, Pollok A, Schaible A, Hinz U, Herfarth C. Multivisceral resection for locally advanced primary colon and rectal cancer: an analysis of prognostic factors in 201 patients. Ann Surg 2002;235(2):217-225

4 Croner RS, Merkel S, Papadopoulos T, Schellerer V, Hohenberger W, Goehl J. Multivisceral resection for colon carcinoma. Dis Colon Rectum 2009;52(8):1381-1386

5 Vignali A, Ghirardelli L, Di Palo S, Orsenigo E, Staudacher C. Laparoscopic treatment of advanced colonic cancer: a case-matched control with open surgery. Colorectal Dis 2013;15(8):944-948

6 Hallet J, Zih FS, Lemke M, Milot L, Smith AJ, Wong CS. Neo-adjuvant chemoradiotherapy and multivisceral resection to optimize R0 resection of locally recurrent adherent colon cancer. Eur J Surg Oncol 2014;40(6):706-712

7 Cukier M, Smith AJ, Milot L, et al. Neoadjuvant chemoradiotherapy and multivisceral resection for primary locally advanced adherent colon cancer: a single institution experience. Eur J Surg Oncol 2012;38(8):677-682

8 Waldron RP, Donovan IA, Drumm J, Mottram SN, Tedman S. Emergency presentation and mortality from colorectal cancer in the elderly. Br J Surg 1986;73(3):214-216

9 Deans GT, Krukowski ZH, Irwin ST. Malignant obstruction of the left colon. Br J Surg 1994;81(9):1270-1276

10 Tan CC, Iftikhar SY, Allan A, Freeman JG. Local effects of colorectal cancer are well palliated by endoscopic laser therapy. Eur J Surg Oncol 1995;21(6):648-652

11 Grund KE, Storek D, Farin G. Endoscopic argon plasma coagulation (APC) first clinical experiences in flexible endoscopy. Endosc Surg Allied Technol 1994;2(1):42-46

12 Eckhauser ML, Mansour EG. Endoscopic laser therapy for obstructing and/or bleeding colorectal carcinoma. Am Surg 1992;58(6):358-363

13 Gainant A. Emergency management of acute colonic cancer obstruction. J Vis Surg 2012;149(1):e3-e10

14 Güenaga KF, Matos D, Wille-Jørgensen P. Mechanical bowel preparation for elective colorectal surgery. Cochrane Database Syst Rev 2011;(9):CD001544

15 Ruggiero R, Sparavigna L, Docimo G, et al. Post-operative peritonitis due to anastomotic dehiscence after colonic resection. Multicentric experience, retrospective analysis of risk factors and review of the literature. Ann Ital Chir 2011;82(5):369-375

16 Dohmoto M, Hünerbein M, Schlag PM. Application of rectal stents for palliation of obstructing rectosigmoid cancer. Surg Endosc 1997;11(7):758-761

17 Cheung HY, Chung CC, Tsang WW, Wong JC, Yau KK, Li MK. Endolaparoscopic approach vs conventional open surgery in the treatment of obstructing left-sided colon cancer: a randomized controlled trial. Arch Surg 2009;144(12):1127-1132

18 van Hooft JE, Bemelman WA, Oldenburg B, et al; collaborative Dutch Stent-In study group. Colonic stenting versus emergency surgery for acute left-sided malignant colonic obstruction: a multicentre randomised trial. Lancet Oncol 2011;12(4):344-352

19 Pirlet IA, Slim K, Kwiatkowski F, Michot F, Millat BL. Emergency preoperative stenting versus surgery for acute left-sided malignant colonic obstruction: a multicenter randomized controlled trial. Surg Endosc 2011;25(6):1814-1821

20 Ho KS, Quah HM, Lim JF, Tang CL, Eu KW. Endoscopic stenting and elective surgery versus emergency surgery for left-sided malignant colonic obstruction: a prospective randomized trial. Int J Colorectal Dis 2012;27(3):355-362

21 Alcántara M, Serra-Aracil X, Falcó J, Mora L, Bombardó J, Navarro S. Prospective, controlled, randomized study of intraoperative 
colonic lavage versus stent placement in obstructive left-sided colonic cancer. World J Surg 2011;35(8):1904-1910

22 Ghazal AH, El-Shazly WG, Bessa SS, El-Riwini MT, Hussein AM. Colonic endolumenal stenting devices and elective surgery versus emergency subtotal/total colectomy in the management of malignant obstructed left colon carcinoma. J Gastrointest Surg 2013; 17(6):1123-1129

23 Van den Berg MW, Ledeboer M, Dijkgraff MG, Fockens P, ter Borg F, van Hooft JE. Long-term results of palliative stent placement for acute malignant colonic obstruction. Surg Endosc 2015;29(6):1580-1585

24 Quereshy FA, Poon JT, Law WL. Long-term outcome of stenting as a bridge to surgery for acute left-sided malignant colonic obstruction. Colorectal Dis 2014;16(10):788-793
25 Poultsides GA, Servais EL, Saltz LB, et al. Outcome of primary tumor in patients with synchronous stage IV colorectal cancer receiving combination chemotherapy without surgery as initial treatment. J Clin Oncol 2009;27(20):3379-3384

26 Anwar MA, D'Souza F, Coulter R, Memon B, Khan IM, Memon MA. Outcome of acutely perforated colorectal cancers: experience of a single district general hospital. Surg Oncol 2006;15(2):91-96

27 Biondo S, Kreisler E, Millan M, et al. Differences in patient postoperative and long-term outcomes between obstructive and perforated colonic cancer. Am J Surg 2008;195(4):427-432

28 Zielinski MD, Merchea A, Heller SF, You YN. Emergency management of perforated colon cancers: how aggressive should we be? J Gastrointest Surg 2011;15(12):2232-2238 